PROCEEDINGS OF THE

AMERICAN MATHEMATICAL SOCIETY

Volume 128, Number 10, Pages 2875-2878

S 0002-9939(00)05366-1

Article electronically published on April 28, 2000

\title{
A REFINEMENT OF THE TORAL RANK CONJECTURE FOR 2-STEP NILPOTENT LIE ALGEBRAS
}

\author{
PAULO TIRAO
}

(Communicated by Roe Goodman)

\begin{abstract}
It is known that the total (co)-homoloy of a 2-step nilpotent Lie algebra $\mathfrak{g}$ is at least $2^{|\mathfrak{z}|}$, where $\mathfrak{z}$ is the center of $\mathfrak{g}$. We improve this result by showing that a better lower bound is $2^{t}$, where $t=|\mathfrak{z}|+\left[\frac{|v|+1}{2}\right]$ and $v$ is a complement of $\mathfrak{z}$ in $\mathfrak{g}$. Furthermore, we provide evidence that this is the best possible bound of the form $2^{t}$.
\end{abstract}

\section{INTRODUCTION}

An outstanding conjecture, known as the Toral Rank Conjecture (TRC) claims that for any nilpotent Lie algebra $\mathfrak{g}$ (over $\mathbf{R}$ or $\mathbf{C}$ ) the total (co)-homology, with trivial coefficients, satisfies the inequality

$$
\left|\mathrm{H}_{*}(\mathfrak{g})\right| \geq 2^{|\mathfrak{z}|},
$$

where $\mathfrak{z}=\operatorname{center}(\mathfrak{g})$.

The TRC is due to S. Halperin ([5], 1987). In 1988, Deninger and Singhof [4] proved it for 2-step nilpotent Lie algebras. Besides this class, a few special cases have been added recently. For example, it was shown in [2] that the TRC holds for $\mathfrak{g}$ if its center has dimension $\leq 5$ or has codimension $\leq 7$.

It turns out that, in general, $2^{|\mathfrak{z}|}$ is quite a lot smaller than $\left|\mathrm{H}_{*}(\mathfrak{g})\right|$, especially when $|\mathfrak{z}|$ is comparatively small compared to $|\mathfrak{g}|$.

In this short note we give a new lower bound for $\left|\mathrm{H}_{*}(\mathfrak{g})\right|$, when $\mathfrak{g}$ is a 2-step nilpotent Lie algebra, that involves both the dimension of the center of $\mathfrak{g}$ and its codimension. Furthermore, by using existing calculations, we show that actually it is the best possible general lower bound of the form $2^{t}$.

Precisely, we give a direct proof of the following

Theorem. Let $\mathfrak{g}$ be a 2-step nilpotent Lie algebra of finite dimension over $\mathbf{R}$ or C. Let $v$ be any direct complement, as vector spaces, of $\mathfrak{z}=\operatorname{center}(\mathfrak{g})$. Then $\mathbf{1}$

$$
\left|\mathrm{H}_{*}(\mathfrak{g})\right| \geq 2^{|\mathfrak{z}|+\left[\frac{|v|+1}{2}\right]} .
$$

Received by the editors August 24, 1998 and, in revised form, November 22, 1998.

2000 Mathematics Subject Classification. Primary 17B56, 17B30.

Key words and phrases. Homology of Lie algebras, 2-step nilpotent Lie algebras, toral rank conjecture.

${ }^{1}$ The right brackets denote the integral part. 


\section{The PRoOF OF THE TheOREM}

We will make use of the following combinatorial result.

Lemma. Let $n$ be a positive integer. Then,

$$
\left|\sum_{j=0}(-1)^{j}\left(\begin{array}{c}
n \\
2 j
\end{array}\right)\right|+\left|\sum_{j=0}(-1)^{j}\left(\begin{array}{c}
n \\
2 j+1
\end{array}\right)\right|=2^{\left[\frac{n+1}{2}\right]} .
$$

Proof. Let $P=\sum_{j=0}(-1)^{j}\left(\begin{array}{c}n \\ 2 j\end{array}\right)$ and $Q=\sum_{j=0}(-1)^{j}\left(\begin{array}{c}n \\ 2 j+1\end{array}\right)$. Since $(1+i)^{n}=P+i Q$ and $(1-i)^{n}=P-i Q$, then $P=\frac{(1+i)^{n}+(1-i)^{n}}{2}$ and $Q=i^{-1} \frac{(1+i)^{n}-(1-i)^{n}}{2}$. Therefore,

$$
|P|=\left\{\begin{array}{lll}
2^{n / 2}, & \text { if } n \equiv 0 \quad \bmod 4 ; \\
2^{(n-1) / 2}, & \text { if } n \equiv 1 \quad \bmod 4 ; \\
0, & \text { if } n \equiv 2 \bmod 4 ; \\
2^{(n-1) / 2}, & \text { if } n \equiv 3 \bmod 4 ;
\end{array} \text { and }|Q|=\left\{\begin{array}{lll}
0, & \text { if } n \equiv 0 \bmod 4 \\
2^{(n-1) / 2}, & \text { if } n \equiv 1 \bmod 4 \\
2^{n / 2}, & \text { if } n \equiv 2 \bmod 4 \\
2^{(n-1) / 2}, & \text { if } n \equiv 3 \bmod 4
\end{array}\right.\right.
$$

from which the lemma follows.

Proof of the Theorem. The homology of $\mathfrak{g}$ is the homology of the Koszul complex $(\wedge \mathfrak{g}, \partial) 2$ that

Put $\mathfrak{g}=v \oplus \mathfrak{z}$. Hence, we can write $\bigwedge \mathfrak{g}=\bigwedge v \otimes \wedge \mathfrak{z}$. It is straightforward to see

$$
\partial: \bigwedge^{p} v \otimes \bigwedge_{\mathfrak{z}}^{q} \longrightarrow \bigwedge^{p-2} v \otimes \bigwedge^{q+1} \mathfrak{z} .
$$

Therefore, it follows that the complex $(\wedge \mathfrak{g}, \partial)$ is the direct sum of an even and an odd subcomplex, precisely $\left(\bigwedge^{2 p} v \otimes \bigwedge \mathfrak{z}, \partial\right)$ and $\left(\bigwedge^{2 p+1} v \otimes \bigwedge \mathfrak{z}, \partial\right)$. Accordingly, the homology of the Koszul complex is the sum of the homologies of each of the even and odd subcomplexes.

It is well known that if $\left(\mathcal{C}=\left(C_{i}\right), \partial\right)$ is a finite complex of finite dimensional vector spaces, then

$$
\left|\mathrm{H}_{*}(\mathcal{C})\right| \geq\left|\sum_{i}(-1)^{i} \operatorname{dim}\left(C_{i}\right)\right|
$$

By applying this to each of the even and odd subcomplexes we get that

$$
\begin{aligned}
\left|\mathrm{H}_{*}(\mathfrak{g})\right| & \geq\left|\sum_{j=0}(-1)^{j} \operatorname{dim}\left(\bigwedge^{2 j} v \otimes \bigwedge \mathfrak{z}\right)\right|+\left|\sum_{j=0}(-1)^{j} \operatorname{dim}\left(\bigwedge^{2 j+1} v \otimes \bigwedge \mathfrak{z}\right)\right| \\
& =2^{|\mathfrak{z}|}\left|\sum_{j=0}(-1)^{j}\left(\begin{array}{c}
|v| \\
2 j
\end{array}\right)\right|+2^{|\mathfrak{z}|}\left|\sum_{j=0}(-1)^{j}\left(\begin{array}{c}
|v| \\
2 j+1
\end{array}\right)\right| .
\end{aligned}
$$

Now the Theorem follows from the Lemma.

Remark. The bound obtained in the Theorem is the best possible, of the form $2^{t}$ ( $t$ an integer), that can be given in general. In fact, among the few available computations there are examples that support this claim.

$$
{ }^{2} \partial\left(x_{1} \wedge \ldots \wedge x_{p}\right)=\sum_{i<j}(-1)^{i+j+1}\left[x_{i}, x_{j}\right] \wedge x_{1} \wedge \ldots \wedge \widehat{x_{i}} \wedge \ldots \wedge \widehat{x_{j}} \wedge \ldots \wedge x_{p}
$$


Example 1. Let $\mathfrak{g}_{2}$ denote the Lie algebra with basis $\left\{x_{1}, x_{2}, y_{1}, y_{2}, z\right\}$ and nonzero brackets $\left[z, x_{i}\right]=y_{i}$ for each $1 \leq i \leq 2$. It was shown in [1] that

$$
b_{0}=1, \quad b_{1}=3, \quad b_{2}=6, \quad b_{3}=6, \quad b_{4}=3, \quad b_{5}=1,
$$

where $b_{i}=\left|\mathrm{H}^{i}\left(\mathfrak{g}_{2}\right)\right|$. Therefore, the total cohomology of $\mathfrak{g}_{2}$ is equal to 20 and our bound is $2^{2+2}=16$.

Example 2. Let $\mathfrak{g}$ denote the Lie algebra with basis $\{a, b, c, d, e, f, g\}$ and non-zero brackets $[a, b]=e,[b, d]=g,[c, d]=e$ and $[a, c]=f$. This Lie algebra appears as $3,7_{D}$ in Seeley's classification [6]. In [3] one can explicitly find that

$b_{0}=1, \quad b_{1}=4, \quad b_{2}=11, \quad b_{3}=14, \quad b_{4}=14, \quad b_{5}=11, \quad b_{6}=4, \quad b_{7}=1$,

where $b_{i}=\left|\mathrm{H}^{i}(\mathfrak{g})\right|$. Hence $\left|\mathrm{H}^{*}(\mathfrak{g})\right|=60$, while our bound is $2^{3+2}=32$.

Example 3. We consider here a family of examples. For each $r \geq 2$ let $E$ be an $r$-dimensional vector space; then $\mathfrak{g}_{r}=E \oplus \bigwedge^{2} E$ is the rank $r$ 2-step free nilpotent Lie algebra. For $e, f \in E,[e, f]=e \wedge f$, these being all the non-zero brackets.

Their homology has been computed by Sigg [7]. Using his result one can compute, for each $r$, the total homology for the rank $r$ algebra. With a simple computer program written in Maple $\mathrm{V}$ we have done it for small $r$ 's. On the other hand, it is clear that our bound is $2^{\left(\begin{array}{r}r \\ 2\end{array}\right)+\left[\frac{r+1}{2}\right]}$. In the following table we show the results for $2 \leq r \leq 13 .\left(t=\left(\begin{array}{c}r \\ 2\end{array}\right)+\left[\frac{r+1}{2}\right]\right)$

\begin{tabular}{|c|c|c|c|c|}
\hline$r$ & $\left|\mathfrak{g}_{r}\right|$ & Total homology & $t$ & $2^{t}$ \\
\hline 2 & 3 & 6 & 2 & 4 \\
\hline 3 & 6 & 36 & 5 & 32 \\
\hline 4 & 10 & 420 & 8 & 256 \\
\hline 5 & 15 & 9800 & 13 & 8192 \\
\hline 6 & 21 & 452760 & 18 & 262144 \\
\hline 7 & 28 & $4.1835024 \times 10^{7}$ & 25 & $3.3554432 \times 10^{7}$ \\
\hline 8 & 36 & $7.691667984 \times 10^{9}$ & 32 & $4.294967296 \times 10^{9}$ \\
\hline 9 & 45 & $2.828336198688 \times 10^{12}$ & 41 & $2.199023255552 \times 10^{12}$ \\
\hline 10 & 55 & $2.073619375892064 \times 10^{15}$ & 50 & $1.125899906842624 \times 10^{15}$ \\
\hline 11 & 66 & $3.040584296923128384 \times 10^{18}$ & 61 & $2.305843009213693952 \times 10^{18}$ \\
\hline 12 & 78 & $8.898500292240756664896 \times 10^{21}$ & 72 & $4.722366482869645213696 \times 10^{21}$ \\
\hline 13 & 91 & $5.2084270468105185237918848 \times 10^{25}$ & 85 & $3.8685626227668133590597632 \times 10^{25}$ \\
\hline
\end{tabular}

\section{ACKNOWLEDGMENTS}

We thank the referee for his illuminating comments.

\section{REFERENCES}

[1] Armstrong G., Cairns G. and Jessup B., Explicit Betti numbers for a family of nilpotent Lie algebras, Proc. Amer. Math. Soc. 125 (1997), 381-385. MR 97d:17013

[2] Cairns G. and Jessup B., New bounds on the Betti numbers of nilpotent Lie algebras, Comm. in Algebra 25 (1997), 415-430. MR 98a:17032

[3] Cairns G., Jessup B., and Pitkethly J., On the Betti numbers of nilpotent Lie algebras of small dimension, Prog. in Math.: Integrable systems and foliations (Albert C. et al., eds.), vol. 145, Birkhauser, 1997, pp. 19-31. MR 98c:17018

[4] Deninger Ch. and Singhof W., On the cohomology of nilpotent Lie algebras, Bull. Soc. Math. France 116 (1988), 3-14. MR 90c:17023 
[5] Halperin S., Le complexe de Koszul en algèbre et topologie, Ann. Inst. Fourier 37 (1987), 77-97. MR 89d:55040

[6] Seeley C., 7-dimensional nilpotent Lie algebras, Trans. Amer. Math. Soc. 335 (1993), 479-496. MR 93d:17015

[7] Sigg S., Laplacian and homology of free 2-step nilpotent Lie algebras, J. Algebra 185 (1996), 144-161. MR 97i:17014

International Centre for Theoretical Physics (ICTP), Trieste, Italy

Facultad de Matemática, Astronomía y Física, Córdoba, Argentina

Current address: Heinrich-Heine-Universität, Mathematisches Institut, 40225 Düsseldorf, Germany

E-mail address: ptirao@bart.cs.uni-duesseldorf.de

E-mail address: Paulo.Tirao@FamaF.uncor.edu.ar 